\title{
Annotazioni sul testo del Salmo 17
}

\author{
Notes on the Text of Psalm 17
}

\author{
STANISŁAW BAZYLIŃSKI \\ Pontificia Facoltà Teologica San Bonaventura, Roma \\ bazylinski@biblico.it \\ ORCID: 0000-0002-1851-9519
}

\begin{abstract}
On the basis of the Hebrew manuscripts and other ancient textual witnesses, this article singles out and discusses many text-critical and translational issues regarding Psalm 17, dwelling particularly upon vv.11 and 14. For v.11, the author accepts the conjec-

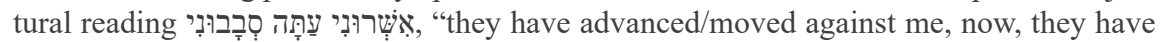

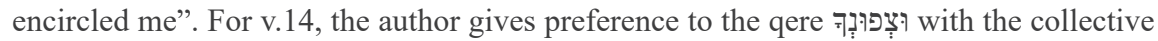
meaning: "and your protected ones".
\end{abstract}

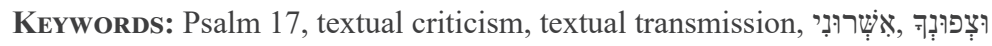

Parole Chiave: Salmo 17, critica testuale, trasmissione del testo,

e difficoltà testuali, che questa supplica individuale presenta, hanno attiraLto da tempo l'attenzione degli interpreti ${ }^{1}$. Benché essi non abbiano raggiunto un consenso sulla maniera di risolverle, i loro contributi filologici soggiacciono al presente articolo che ripercorrerà, seguendo l'ordine dei versetti, i principali aspetti della trasmissione del testo e dei punti controversi. Partiamo dall'ultima traduzione ufficiale della CEI (2008) per renderci conto delle soluzioni alternative in grado di contribuire ad una maggiore comprensione del Sal $17^{2}$. Piuttosto che

1 Si vedano, ad es., E.J. Kissane, «Some Critical Notes on Psalm XVII», Bib 9 (1928) 89-96; J. Lindblom, «Bemerkungen zu den Psalmen I», ZAW 59 (1942-1943) 1-13, spec. 7-13; J. Leveen, «The Textual Problems of Psalm XVII», VT 11 (1961) 48-54; J. van der Ploeg, «Le psaume xvii et ses problèmes», כה 1940-1965 (OTS 14; Leiden: Brill 1965) 273-295, spec. 278-293; D. Barthélemy et al., Critique textuelle de l'Ancien Testament. IV. Psaumes (OBO 50/4; Fribourg: Academic Press - Göttingen: Vandenhoeck \& Ruprecht 2005) 71-78; Á. Aparicio Rodríguez, Comentario filológico a los Salmos y al Cantar de los Cantares (Madrid: Biblioteca de Autores Cristianos 2012) 104-110.

2 Per lo status quaestionis relativo alla datazione, alla Vorlage, al carattere delle traduzioni antiche e alla relazione tra di esse, si veda The Hebrew Bible. I/C. Writings (a cura di A. Lange - E. Tov) (THB 1; Leiden - Boston: Brill 2017) 5-147; per le versioni antiche usate nel corso di questo articolo, segnaliamo i seguenti contributi: B.A. Strawn, «Textual History of Psalms», 5-23; J. Smith, «Septuagint», 82-88; D.M. Stec, «Targum», 88-93; I. Carbajosa, «Peshitta», 93-98; J.M. Meade, «Hexaplaric Greek Translations», 98-103; J. Rogers, «Vulgate», 104-110; J.M. Cañas Reíllo, «Vetus Latina», 115-119. 
riproporre sic et simpliciter i dati ormai acquisiti dalla ricerca biblica, abbiamo preferito discutere e delineare, inter alia, alcune di tali soluzioni.

\section{Versetto 1}

Ascolta, Signore, la mia giusta causa, sii attento al mio grido.

Porgi l'orecchio alla mia preghiera: sulle mie labbra non c'è inganno.

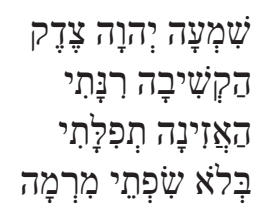

\section{1. צִ צִ}

La lezione masoretica צֶֶֶ, «giustizia», è confermata da Simmaco (Sym) e Te-

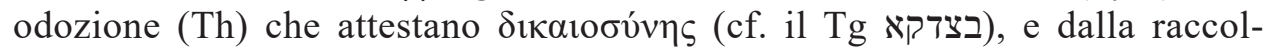
ta dei mss ebraici che non presenta altre varianti ${ }^{3}$. I LXX leggono, invece,

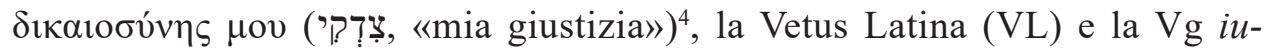

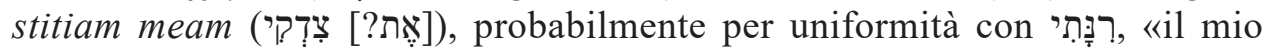

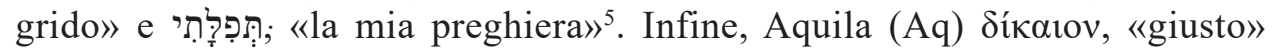
e il salterio iuxta Hebraeos (Hier) ${ }^{6}$ iustum leggono secondo il metodo della retroversione צִדְדיק.

\section{2. בִלִ}

Le traduzioni della preposizione (לדּ (rivelano invece una certa varietà ${ }^{7}$. I LXX ( $\dot{\varepsilon} v)$, la VL e la Vg (in) fanno pensare a un valore locale di ঙ̣:, «sulle

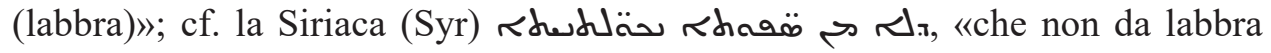

3 Cf. Kennicott, II, 317.

4 Questa variante è influenzata forse da אֶלהּי צִדְקָ, «Dio della mia giustizia», del Sal 4,2. Si confronti

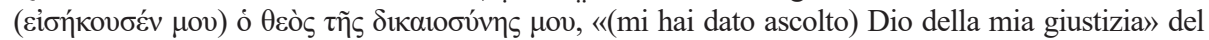

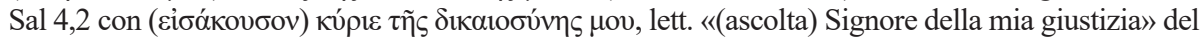
Sal 16(17),1.

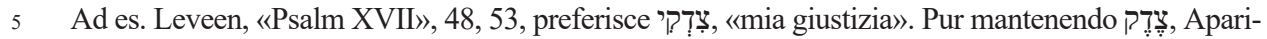

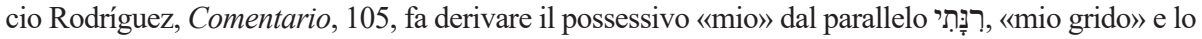
considera un caso «del doble uso del sufijo». Invece, Van der Ploeg, «Le psaume xvii», 278-279,

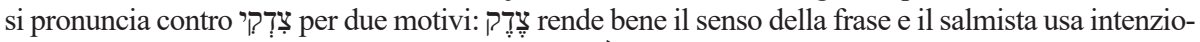

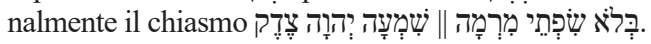

6 Per le versioni latine, si fa riferimento a: P. Sabatier (a cura di), Bibliorum sacrorum latinae versiones antiquae seu Vetus Italica (Parisiis: apud Franciscum Didot 1751) II; Biblia Sacra iuxta latinam Vulgatam versionem ad codicum fidem... cura et studio monachorum abbatiae pontificiae s. Hieronymi in Urbe ordinis sancti Benedicti edita. Liber Psalmorum (Romae: typis Polyglottis Vaticanis 1953) X; H. de Sainte-Marie (a cura di), Sancti Hieronymi Psalterium iuxta Hebraeos (CBLa 11; Roma: Abbaye Saint-Jérôme - Città del Vaticano: Libreria Vaticana 1954).

7 Va sottolineato che essa non dipende dalla trasmissione del testo ebraico, che non presenta varianti; cf. Kennicott, II, 317. 


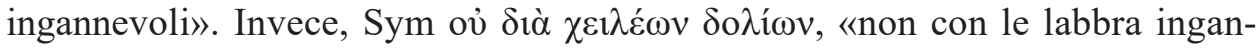
nevoli», fa pensare a un valore strumentale ${ }^{8}$. A nostro avviso, la preposizione $\underset{?}{\mathfrak{P}}$, insieme alla negazione לאיל, mette in scena l'assenza di labbra ingannevoli (cf. Hier absque; il Tg בדלא, «senza») ${ }^{10}$. La frase potrebbe essere tradotta alla lettera: «senza labbra d'inganno» o più liberamente «preghiera [fatta/formulata] con labbra prive d'inganno», «preghiera non [fatta/formulata] con labbra d'inganno».

\section{Versetto 2}

Dal tuo volto venga per me il giudizio, i tuoi occhi vedano la giustizia.

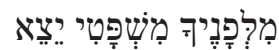

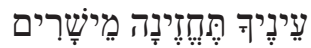

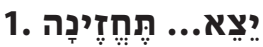

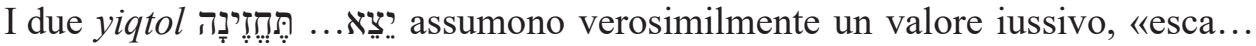

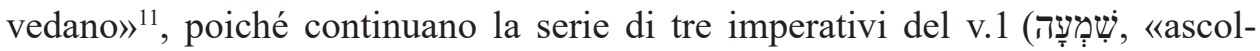

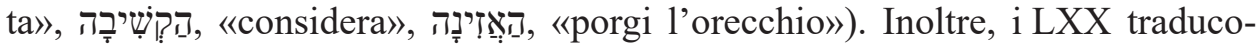
no, rispettivamente, con l'ottativo $\dot{\varepsilon} \xi \dot{\varepsilon} \lambda \theta$ ol, «proceda» (ott. aor. att. di $\dot{\varepsilon} \xi \dot{\varepsilon} \rho \chi 0 \mu \alpha$ )

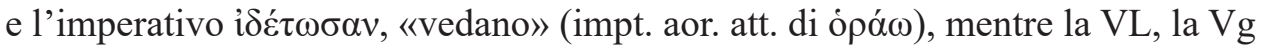
e Hier con i congiuntivi prodeat e videant.

\section{2. עַינֶיך}

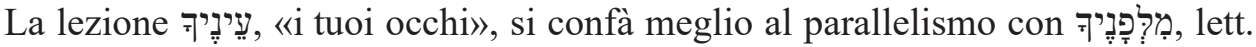
«dal tuo volto»e trova il sostegno dei mss ebraici ${ }^{12}$ e dei LXX mss A, 187, 210

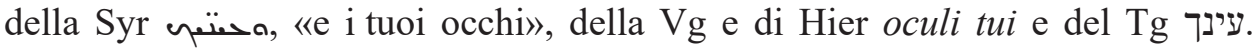

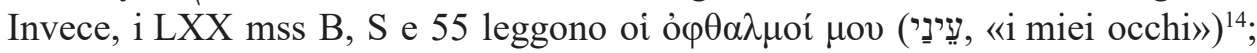
cf. la VL oculi mei.

8 HALAT, 487: «m[it]. Lippen ohne Trug», «con le labbra senza/libere dall'inganno».

9 Cf. Nm 35,22.23; Ger 22,13; Ez 22,29; Sal 44,13; Gb 8,11; 30,28; Pro 13,23; 16,8; 19,2; Lam 1,6; $1 \mathrm{Cr} 12,18.34 ; 2 \mathrm{Cr} 21,20$.

10 E. Jenni, Die hebräische Präpositionen. I. Die Präposition Beth (Stuttgart-Berlin - Köln: Kohlhammer 1992) 357 ( $n^{\circ}$ 5431), rende: «ohne trügerische Lippen», riconducendo il Sal 17,1 alla rubrica 54: «Nominalisierung eines Nichtexistenz-Satzes» e commentando: «Die Bezeichnung zwischen den parallelisierten Satzinhalten kann auch hier mit „,indem“ (so wohl Jes 57,1; Ez 38,11; Ps 17,1; Hi 7,6)».

11 Contro, ad es., E. Podechard, Le Psautier. Notes critiques. I. Psaumes 1-75 (BFCLL 4; Lyon: Facultés Catholiques 1949) 74.

12 Ad eccezione di tre mss in scriptio defectiva עינך, Kennicott, II, 317, non attesta altre varianti.

13 Cf. R. Holmes - J. Parsons, Vetus Testamentum Graecum cum variis lectionibus (Oxonii: e typographeo Clarendoniano 1823) III, ad locum.

14 Lettura accolta da Leveen, «Psalm XVII», 48, 53. 


\section{3. מִישָׁרִים}

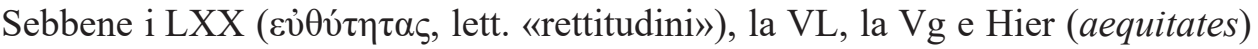
traducano מֶּשָׁרים con il plurale, il singolare andrebbe conservato sulla scorta

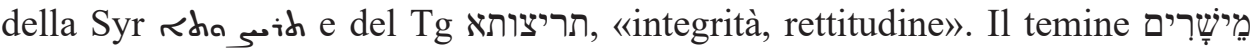
è un pluralia tantum (19x), il cui valore singolare o plurale dipende dai termini messi in parallelo o in serie ${ }^{15}$. La costruzione del v. 2 milita a favore del singolare

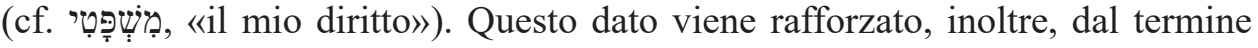
צֶ, «giustizia», che nel v.1 è il primo complemento oggetto richiesto dall'oran-

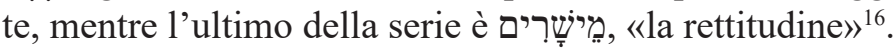

\section{Versetto 3}

Saggia il mio cuore, scrutalo nella notte, provami al fuoco: non troverai malizia. La mia bocca non si è resa colpevole,

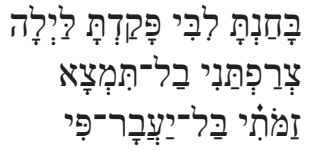

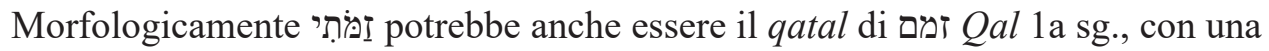
accentuazione anomala (rebia sopra il taw; cf. Dt 32,41; Is 44,16), «mi sono proposto», «ho progettato/escogitato/premeditato (il male)» ${ }^{17}$, oppure l'infinito costrutto Qal (in questo punto con l'accento correttamente collocato) di זמם con suffisso pronominale della 1a sg. ${ }^{18}$, «il mio progettare». In questo secondo caso l'infinito costrutto avrebbe l'accezione nominale de «il mio progetto/piano».

Le stesse versioni sono in disaccordo: il qatal è testimoniato dal Tg (חשבית,

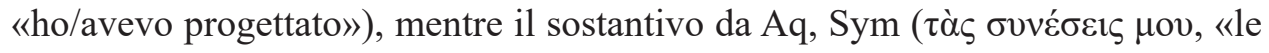
mie conoscenze») e Hier (cogitationes meas), che presuppongono il plurale în,

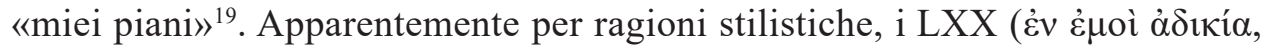

15 Per il singolare, si vedano Is 26,7; 45,19; Sal 9,9; 58,2; 98,9; Pro 1,3; 2,9; 1 Cr 29,17; invece, per il plurale Is 33,15; Pro 8,6; Dn 11,6.

16 Per מִישָׁרים || צֶדָק, si vedano Is 45,19; Sal 58,2; Pro 1,3; 2,9; cf. Sal 9,9; 98,9. Un simile parallelismo è attestato anche tra i termini accadici mïšarum, «giustizia» || kittu, «correttezza/onestà», cf. AHw, 494-495, 659-660; CAD VIII, 470-471, CAD X/2, 117-118.

17 Cf. BL § 58m; R. Meyer, Hebräische Grammatik, 3a ed. (SG 764/764a/764b; Berlin: De Gruyter 1969) II, 145 (\$ 79,2c).

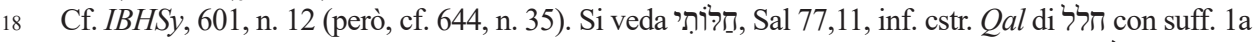

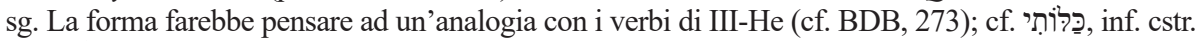
Pi di כלה, Ger 9,15; 49,37; Ez 5,13. C.-F. Houbigant, Notae criticae in universos Veteris Testamenti

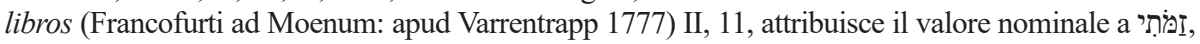

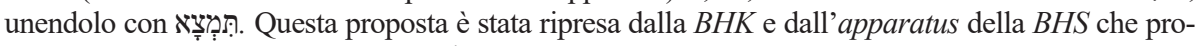

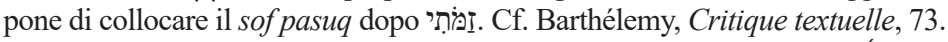

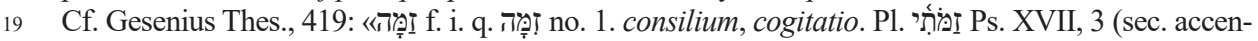

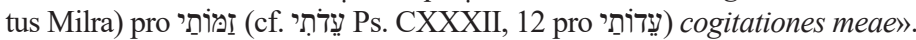


《in me ingiustizia»), la Syr (صه حص), la VL e la Vg (in me iniquitas) leggono

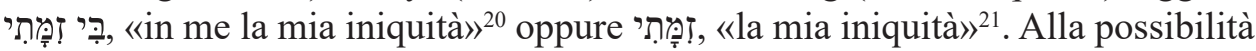
che si tratti di un sostantivo si contrappone la scriptio plena זמותי dei $49 \mathrm{mss}^{22}$, che attestano come seconda vocale la «o». Inoltre, nei mss ebraici non c'è riscontro alla preposizione ?ִ̣. Di conseguenza, le versioni antiche mostrano che inteso come verbo finito, solleva una difficoltà riguardo al senso, richiedendo un complemento oggetto (ad es. malizia, cattiveria) e imponendo di considerare il v.3c come una sorta di frase condizionale ( «Se avessi pensato malizia, non avrebbe attraversato la mia bocca»). Per risolvere la difficoltà, il $\mathrm{Tg}$ ha fatto ricorso a una doppia traduzione con un sostantivo seguito da un verbo: שחיתא חשבית, lett. «colpa avevo progettato».

In conclusione, si può affermare che la proposta masoretica (lett. «il mio pensare», inf. cstr. + suff. 1a sg. $)^{23}$ sia meno problematica rispetto ad altre letture e congetture ${ }^{24}$.

\section{Versetto 4}

secondo l'agire degli uomini; seguendo la parola delle tue labbra, ho evitato i sentieri del violento.

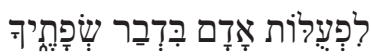

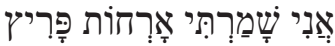

\section{1. לִפעעלוֹת}

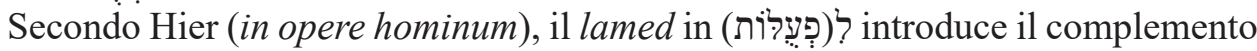

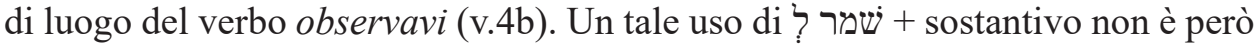

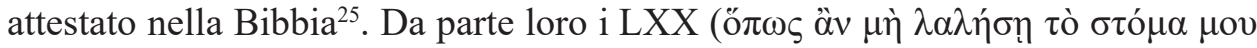

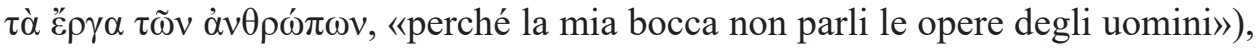
la VL e la Vg (ut non loquatur os meum opera hominum) considerano il lamed

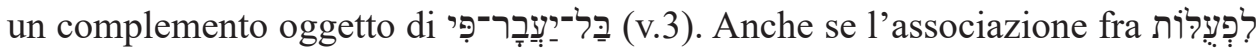

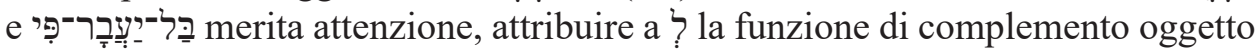
risulta problematico. Infatti,

20 La congettura Țị̦ è appoggiata da HALAT, 262; Ges18, 304.

21 Cf. Schleusner, I, 45.

22 Cf. Kennicott, II, 317.

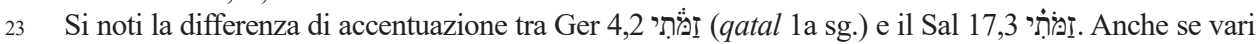
autori ritengono che si tratti di due varianti della stessa forma grammaticale, ci pare che questa forma diversa indichi la difformità grammaticale, come attestano versioni antiche.

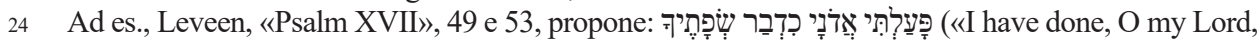
according to the word of thy lips», 54).

25 Cf. complemento oggetto (1Re 8,24.25 || 2Cr 6,15-16; 5,11); complemento di tempo (Ger 3,5; Sal 89,29); complemento oggetto indiretto (Sal 39,2); complemento d'agente (Qo 5,12). 


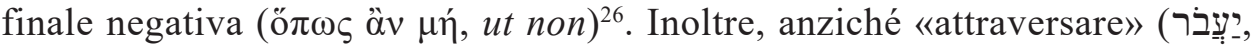
da עבר), le succitate versioni usano il verbo "parlare» ( $\lambda \alpha \lambda \eta \dot{\sigma n}$, loquatur, ebr. דבר Pi), modificando il testo ebraico, seguito invece da Aq e Sym, che tradu-

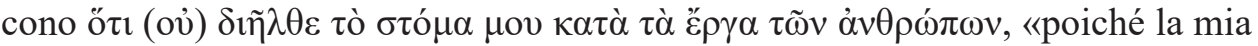
bocca (non) ha seguito - lett. passato/attraversato - secondo le opere degli uomi-

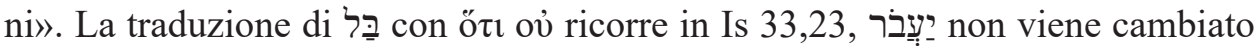

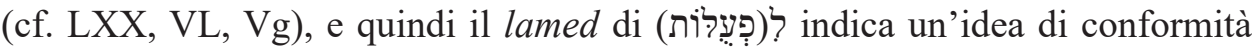
(«in accordo con», «secondo»; cf. Gn 1,11.12.21.24.25; 6,20; 7,14; 8,19; 10,5; Sal 119,154; ecc. $)^{27}$. Ci sembra dunque preferibile questa ultima traduzione.

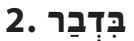

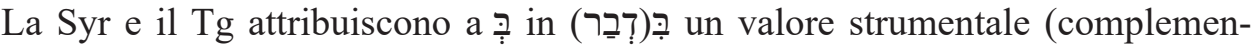

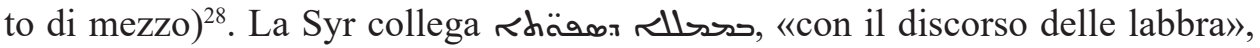
a a del v.3, avendolo già modificato prima (cf. pretazione strumentale del Tg במימר שפוותך, «con la parola delle tue labbra», è stata resa possibile mediante l'aggiunta del verbo אכסנר,, «ho rimproverato»" finalizzata a una maggiore chiarezza stilistica dei vv.3-4.

È più ragionevole seguire i LXX ( $\delta \iota^{\alpha}+$ acc.), la VL, la Vg e Hier (propter) attribuendo il valore causale alla preposizione $\underset{?}{?}$, che esprime un'autorizzazione

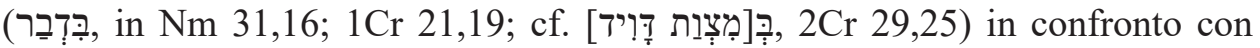

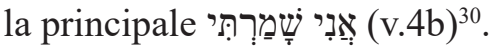

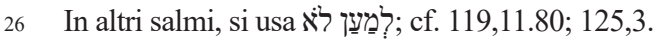

27 Cf. E. Jenni, Die hebräische Präpositionen. III. Die Präposition Lamed (Stuttgart - Berlin - Köln: Kohlhammer 2000) 281 ( $n^{\circ}$ 9315), che distingue una relazione normativa («Normative Relationen»), introdotta dal lamed. Invece, A.F. Kirkpatrick, The Book of Psalms. I. Psalms I-XLI (CBSC; Cambridge: University Press 1906) 80; C.A. Briggs - E.G. Briggs, A Critical and Exegetical Commentary on the Book of Psalms (ICC; Edinburgh: Clark 1907) I, 134; T. Lorenzin, I Salmi. Nuova versione, introduzione e commento (I Libri Biblici. Primo Testamento 14; Milano: Paoline 2000) 85; N.A. van Uchelen, Psalmen. I. 1-40, 3 ed. (POuT; Nijkerk: Callenbach 1986) 106, e altri autori ipotizzano che questa preposizione introduca il casus pendens («per quanto riguarda», «quanto a»). Ciò è messo fortemente in dubbio da GK $\S 143 \mathrm{~h}$.

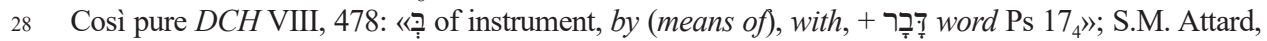
«From Well-fed Lions to Sitting Ducks. A Study of Complex Metaphors in Psalm 17», Networks of Metaphors in the Hebrew Bible (a cura di D. Verde - A. Labahn) (BETL 309; Leuven - Paris - Bristol, CT: Peeters 2020) 180: «with», «by».

29 Cf. L. Díez Merino (a cura di), Targum de Salmos. Edición Príncipe del Ms. Villa-Amil n. 5 de Alfonso de Zamora (Bibliotheca Hispana Bíblica 6; Madrid: Consejo Superior de Investigaciones Científicas 1982) 87; D.M. Stec (a cura di), The Targum of Psalms (ArBib 16; London - New York: Clark 2004) 47: «I have rebuked》.

30 Jenni, Präpositionen, I, 355 ( $\mathrm{n}^{\circ}$ 5211). Alla luce di quanto detto in corpore, ci discostiamo da Attard,

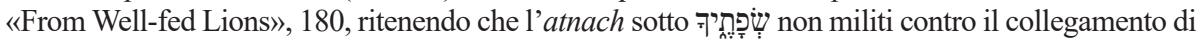

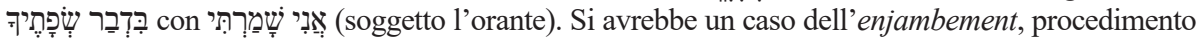
stilistico ben attestato nel Salterio (cf. 5,8; 35,27; 40,5; 55,18b-19a; 69,2; 101,7b; 116,1.2.5.7.8.9; 132,1.2.4.10,11b.12b; ecc.) e nel Sal 17,7.8-9.13-14. 


\section{3.}

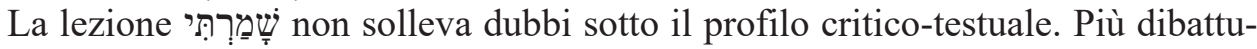

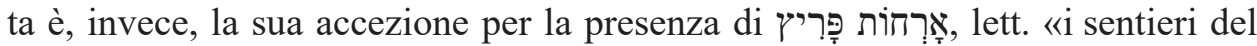
violento» che, insinuando apparentemente il significato di «custodire/mantenere le vie del violento», si attaglia poco al contesto immediato. Per risolvere questa contraddizione, alcune versioni antiche modificano il testo ebraico. In luogo di

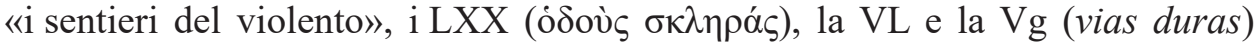

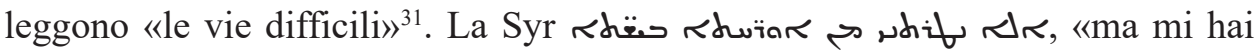
custodito dalle vie cattive», attribuisce a Dio, e non all'orante, la funzione di soggetto del verbo «custodire» e cambia altresì il sostantivo «violento» nell'aggetti-

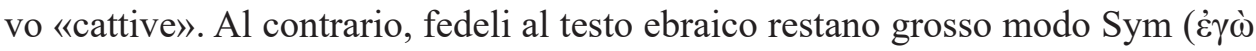

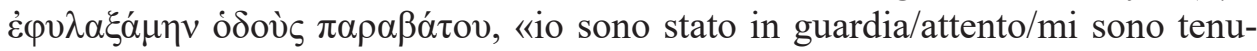
to lontano/guardato dalle vie del violatore/trasgressore»), Hier (ego observavi vias latronis) e il Tg (אנא נטרית ארחת חציפא, «ho osservato/sorvegliato i sentieri dell'arrogante/sfacciato»). Alla luce di queste traduzioni, non risulta giustificato il cambiamento di פִִּ (LXX, Syr, VL, Vg) e/o del suo significato ${ }^{32}$.

Per risolvere le difficoltà di traduzione è preferibile attribuire a שֵָָׁ un'accezione diversa da «custodire, mantenere». Hier e il Tg suggeriscono quella di «osservare», «guardare», nel senso di «vigilare», «tenere a bada» o «sorvegliare».

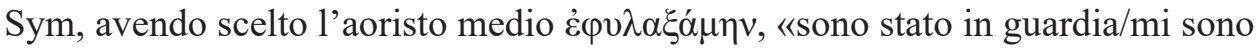
tenuto lontano», mette in risalto il distacco dell'orante dalle azioni di una persona cattiva. Nell'Antico Testamento, la costruzione שמר + complemento oggetto ${ }^{33}$

31 Lezione preferita da Aparicio Rodríguez, Comentario, 107: «senderos abruptos».

32 Ad es., in base all'etimologia accadica e araba, G.R. Driver, «The Root פרץ in Hebrew», JThS 25 (1924) 177-178, cerca di mostrare un doppio significato della radice פרץ mand». Questa etimologia è stata mutuata da qualche commentatore e lessicografo; ad es., Kissane, «Psalm XVII», 90, unisce le ultime due parole del v.4 con il v.5 e modifica il testo ebraico:

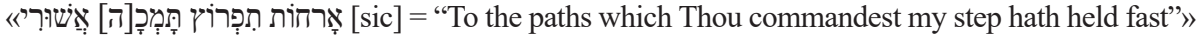
(91). Il ricorso all'accadico e all'arabo è stato incluso in Zorell, 668: "“vias hominis violenti (= impii)" observavi = cavi (at frt. in 'פ latet hic subst. cui respondet ass. parșu obligatio religiosa [AHw, 835: «Amt; Kult(ordnung)»], ar. fard praeceptum, et verti potest “servavi vias praecepti”» e in DCH VI, 769, che ha proposto per

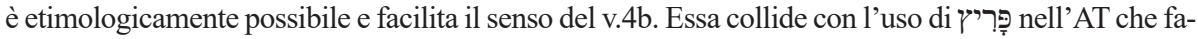
vorisce piuttosto l'accezione di «violento», «ladro» (cf. Is 35,9; Ger 7,11; Ez 7,22; 18,10; Dn 11,14), attestata da Aq, Sym, Hier e Tg.

Da parte sua, J.J. Glück, «Das Verb PRȘ in the Bible and in the Qumran Literature», RdQ 5 (1964-65) 125 e n. 23, ritiene che il significato base di פָּרָּר sia «landowner», «leader», riscontrabile in Dn 11,4 [sic] e nel Sal 17,4. In seguito, פָּרָּר avrebbe acquistito una connotazione negativa indicando una persona violenta (cf. Ez 7,22), un fuorilegge (cf. Ger 7,11 e 1Sam 25,10). A nostro avviso, il contesto dei vv.1-3.5 non depone a favore del significato di «proprietario», «guida», poiché l'orante sta proclamando la propria innocenza dinanzi agli altri. Quindi è più appropriato qui il significato negativo; cf. C. Toll, «Die Wurzel PRṢ in Hebräischen», OrSuec 21 (1972) 83; P.J. Harland, «Robber or Violent Man? A Note on the Word pārîș», VT 46 (1996) 533.

33 Meno pertinente, a nostro parere, è la costruzione שמר מִן, proposta, ad es., da Briggs - Briggs, Psalms, I, 134, «evitare», «guardarsi da», che cita Gs 6,18 e Dt 4,9. 
assume più volte il significato di «tenere d'occhio» (cf. Is 40,20; Sal 37,34.37; 41,$3 ; 56,7 ; 86,2 ; 91,11 ; 107,43 ; 130,3$; ecc.), «scrutare» (Gb 33,11), «vigilare» (cf. 2Sam 11,16), «sorvegliare» (cf. Gn 3,24; 1Sam 19,11 || Sal 59,1), che si adatta al nostro versetto.

\section{Versetto 6}

Io t'invoco poiché tu mi rispondi, o Dio; tendi a me l'orecchio, ascolta le mie parole,

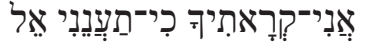

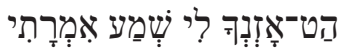

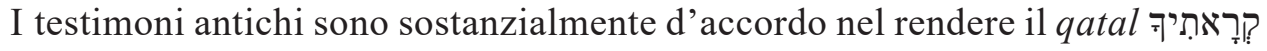

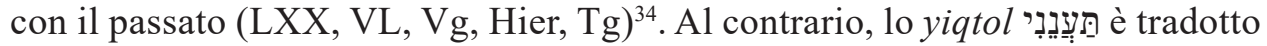
o con il passato (LXX, Syr, VL, Vg) o con il futuro (Hier, Tg). La resa con il futuro è rafforzata dalla costruzione qatal kî yiqtol (cf. Gdc 17,13; Sal 37,13; $140,13)$, benché per lo yiqtol sia ammissibile un valore volitivo nel senso di

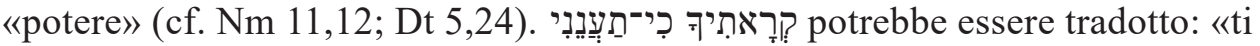
ho chiamato, perché mi rispondi»o «ti ho chiamato, perché [tu] possa rispondermi».

\section{Versetto 7}

mostrami i prodigi della tua misericordia, tu che salvi dai nemici chi si affida alla tua destra.

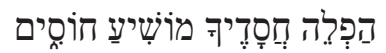

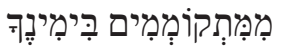

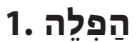

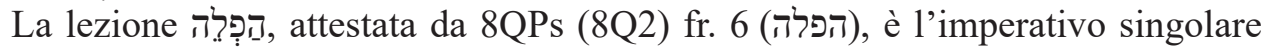
maschile di פלה Hi $H H^{335}$, «far qualcosa di straordinario/ meraviglioso», «far prodigi» (cf. Sym $\pi \alpha \rho \alpha \delta o ́ \xi \alpha \sigma o v$, impt. aor. da $\pi \alpha \rho \alpha \delta o \xi \alpha ́ \zeta \omega$, 《rendere meraviglioso/straordinario») e non di פלה, «separare», «mettere a parte». Quest'ultima accezione è contraddetta anche dai LXX $\theta \alpha v \mu \alpha ́ \sigma \tau \omega \sigma o v$

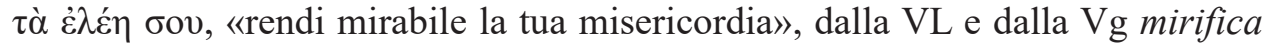
misericordias tuas e da Hier mirabilem fac misericordiam tuam.

34 Van der Ploeg, «Le psaume xvii», 283, ritiene che si tratta del perfetto incoativo: l'orante ha cominciato ad invocare di Dio e continua a farlo; cf. Podechard, Le Psautier, 75.

35 BL § 54r: «orthogr. Angleichung an die Verba וליו" לc. E. König, Hebräisches und aramäisches Wörterbuch zum Alten Testament (Leipzig: Dieterich 1931) 362. In una quarantina di mss di Kennicott, II, 317, si legge הפלא. 


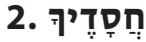

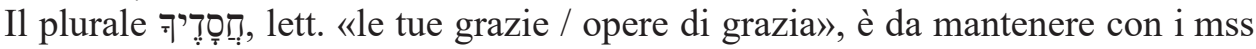
ebraici e con i LXX, la VL, la Vg e il Tg (contro il singolare della Syr e di Hier).

\section{3. מוֹשִיע}

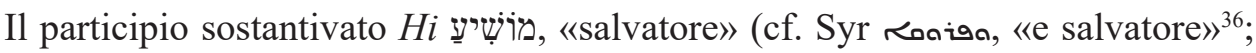
Hier salvator; Tg פריקהון, «loro redentore»), i masoreti l'associano strettamen-

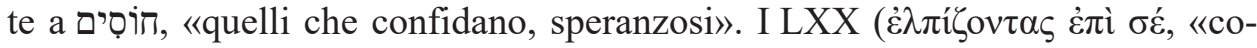
loro che sperano in te») e la Vg (sperantes in te) aggiungono probabilmente il senso della frase; infatti nella Bibbia il sintagma חד: re 35 volte (22x nel Salterio) $)^{37}$, mentre l'uso assoluto di חè attestato soltanto nel Sal 17,7. Il TM, che non riporta le varianti testuali, è confermato dalla Syr (2), "di coloro che sperano») da Hier (sperantium; cf. la VL rectos corde) e dal Tg (דסרי, «di coloro che sperano»).

\section{4וסים}

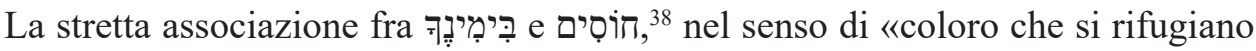

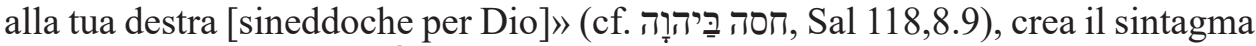

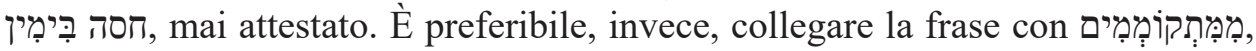
«da quanti insorgono», come confermano i LXX ( $\dot{\varepsilon} \kappa \tau \tilde{\omega} \nu \dot{\alpha} v \theta \varepsilon \sigma \tau \eta \kappa o ́ \tau \omega \nu \tau \tilde{n} \delta \varepsilon \xi 1 \tilde{\alpha}$

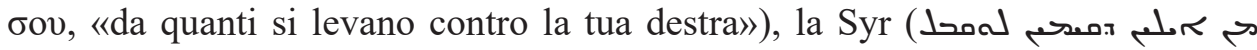
sch, «da coloro che insorgono contro la tua destra»), la VL, la Vg e Hier (a resistentibus dexterae tuae). Le versioni permettono, inoltre, di attribuire alla pre-

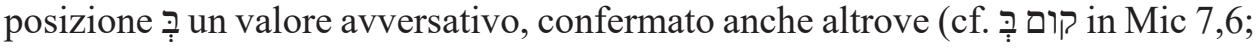
Sal 27,12; Gb 16,8).

\section{Versetto 8}

Custodiscimi come pupilla degli occhi, all'ombra delle tue ali nascondimi,

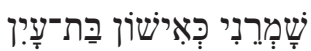

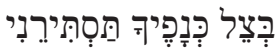

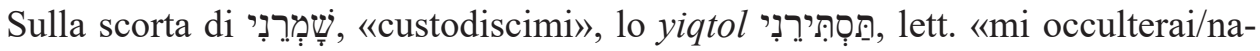
sconderai», equivale ad un imperativo (cf. la VL, i mss F e I della Vg, Hier protege $m e)^{39}$.

\footnotetext{
36 I mss $10 \mathrm{t} 4$ e $12 \mathrm{t} 1$ ommettono il waw iniziale.

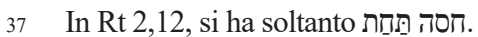

38 Cf. Jenni, Präpositionen, I, 200 (n²323): «sich bergen».

$39 \mathrm{JM} \S 113 \mathrm{~m}$.
} 


\section{Versetto 9}

di fronte ai malvagi che mi opprimono, ai nemici mortali che mi accerchiano.

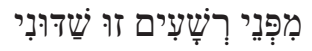

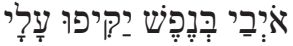

I LXX ( riginale ebraico

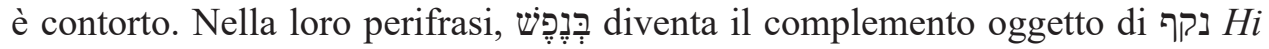
e l'accento cade sul pericolo in cui è incorsa la vita dell'orante («i miei nemici hanno accerchiato la mia anima»). La lettura delle versioni antiche non ha trovato molti consensi nelle traduzioni moderne, poiché darebbe in retroversione

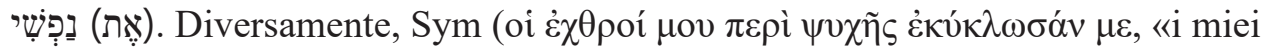
nemici quanto alla vita/a proposito della vita mi circondarono») ricalca pedisse-

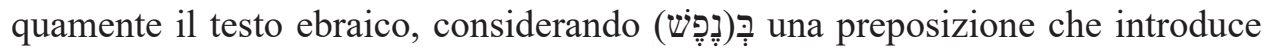
una limitazione ${ }^{40}$. La proposta è stata accolta da qualche lessicografo ${ }^{41}$ e dalle traduzioni moderne che evidenziano la pericolosità dei nemici («mortali», ad es.:

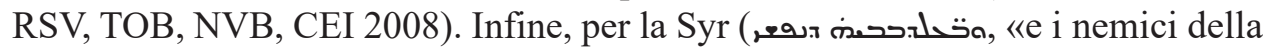

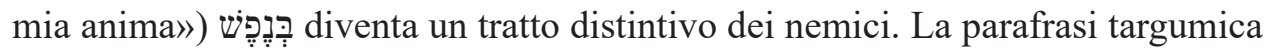
(ברעות נפשיהון), "con il desiderio della loro anima») chiarisce il testo ebraico indirizzando la volontà nemica contro l'orante. Così ritengono anche grammatici e lessicografi moderni ${ }^{42}$.

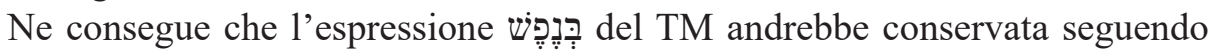
Sym in modo da attribuire a נִפֶֶ il significato di «brama», «animosità», «furore», «accanimento» (cf. רעות del Tg) ${ }^{43}$. La preposizione ᄁ̦i introduce la modalità dell'agire pressante del nemico (נקף Hi), caratterizzata dall'ostilità ${ }^{44}$.

\section{Versetto 10}

Il loro animo è insensibile, le loro bocche parlano con arroganza.

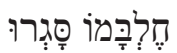

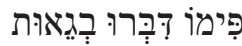

40 Jenni, Präpositionen, I, 344: «Begleitumstand» («circostanza concomitante»).

$41 \quad D C H$ V, 730: «my enemies as regards life, i.e. my mortal enemies»; cf. Aparicio Rodríguez, Comentario, 108: «enemigos mortales».

42 Si vedano le due note successive.

43 Cf. Ez 16,27; 25,6; Sal 27,12; 41,3; cf. BDB, 660: «with greed»; Ges18, 834: «Gier, Begierde, Verlangen»; Stec, The Targum, 48, n. 10: «with greed».

44 Cf. Gesenius Thes., 901: «odium»; HALAT, 673: «'ֶֶ d. Feinde = Hassgien»; Jenni, Präpositionen, I, 347 ( $\left.{ }^{\circ} 4465\right)$ : «Haßgier». 


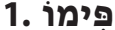

Il sostantivo iפ, «la loro bocca», è un soggetto anticipato (cf. LXX, VL, Vg) ${ }^{45}$ con un suffisso poetico (cf. Sal 58,7; 59,13). La sineddoche con il verbo alla

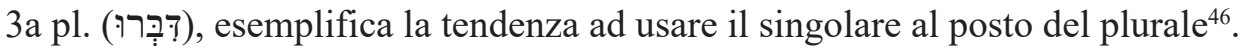

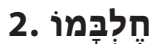

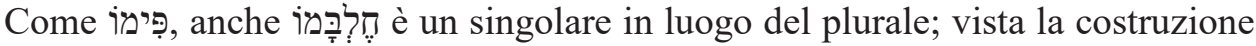

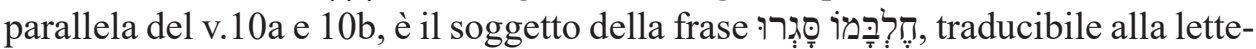
ra: «le loro viscere si sono chiuse»o «il loro grasso si è chiuso». L'uso riflessivo passivo di סגר Qal è attestato da Es 14,3; Gs 2,7; Gdc 3,2247. La chiarezza stilistica, nonostante l'immagine del grasso che si chiude, ha fatto sì che le versioni antiche (LXX, VL, Vg) ${ }^{48}$ e moderne (ad es. RSV, EIN, NVB, CEI 1974) consi-

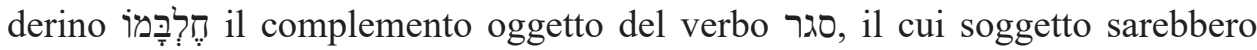
«i miei nemici» del v.9b.

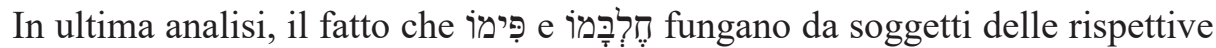
frasi si addice allo stile del salmista che, a più riprese, usa come soggetti alcune

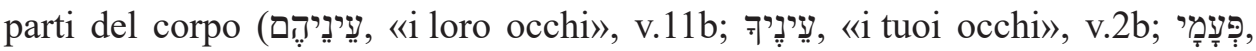
«i miei piedi», v.5b; cf.

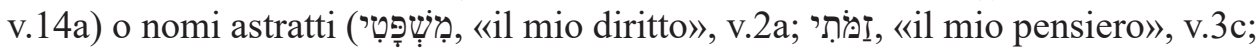

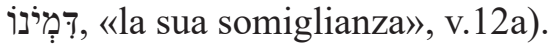

\section{Versetto 11}

Eccoli: avanzano, mi circondano, puntano gli occhi per gettarmi a terra,

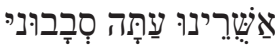

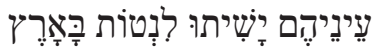

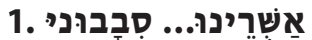

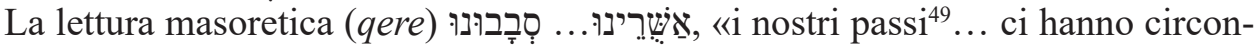

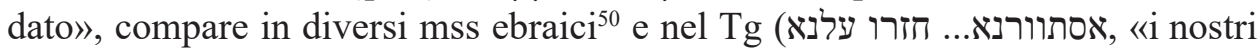
passi... ci hanno circondato»).

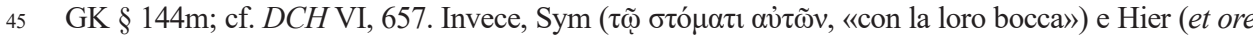

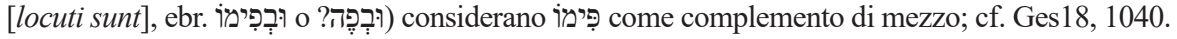

46 Per gli altri esempi, si veda JM § 1361, che traduce il v.10b: «their mouth(s) have said».

47 Nella stessa direzione si muove Sym $\dot{\alpha} \pi \varepsilon \varphi \rho \alpha ́ \gamma \eta \sigma \alpha v$ (ind. aor. pass. di $\dot{\alpha} \pi \circ \varphi \rho \alpha ́ \sigma \sigma \omega)$ ), «(con il loro grasso) si sono chiusi/otturati/sbarrati»; cf. Rashi e Ibn Ezra, che sottintendono la preposizione

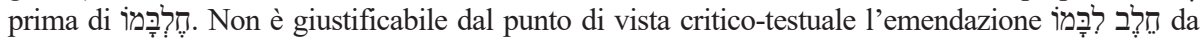
parte di Leveen, «Psalm XVII», 51, 53.

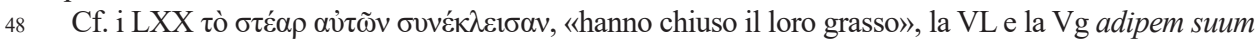
concluserunt, mentre Hier adipe suo concluserunt [animam meam].

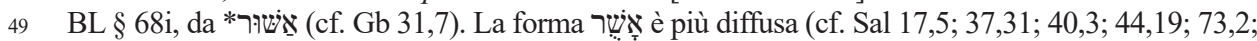
Gb 23,11; Pro 14,15).

50 Cf. Kennicott, II, 317. 
Tenendo presente che i rispettivi emistichi del v.11 sono costruiti in maniera simile (nome-verbo), il TM del v.11a può essere tradotto in due modi: 1) «I nostri passi (soggetto), ora/adesso, ci hanno circondato»; 2) «Ora ci hanno circondato, i nostri passi (complemento oggetto) $\rangle^{51}$. La prima resa è possibile in virtù della continuità con lo stile del salmista che inaugura con i soggetti i rispettivi emistichi del v.10 (cf. supra). Ciononostante, questa resa crea una difficoltà di senso nel v. $11 \mathrm{a}^{52}$.

Sembrerebbe, dunque, più logica la seconda traduzione che individua nei nemici dell'orante (v.9b) il soggetto del verbo סְרָבוּוּנוּ, creando nel v.11a un'amplificazione apposizionale, una specie di epanortosi («ci hanno circondato, [ciò̀] i nostri passi» $)^{53}$. Anche se questo pleonasmo bilancia la lunghezza degli emistichi, esso produce un sintagma mai attestato altrove («circondare i passi di qualcuno») ed è poco utile dal punto di vista del contenuto. Infatti, la frase «ci hanno circon-

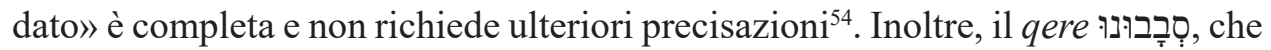
rappresenta una correzione dei masoreti per ristabilire l'ordine sintattico distur-

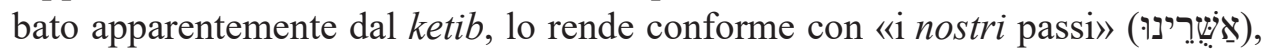
ma non si accorda bene con il resto del salmo, poiché è osteggiato sempre un

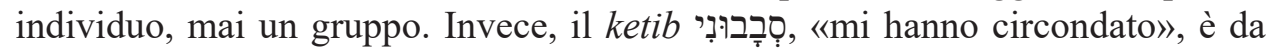

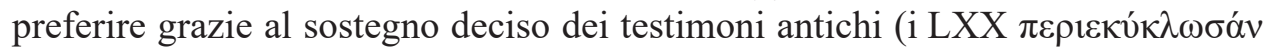
$\mu \varepsilon$, «mi hanno circondato», Sym $\pi \varepsilon \rho 1 \varepsilon \kappa v ́ \kappa \lambda o v v ~ \mu \varepsilon$, «mi circondavano», la Syr حخحمد, «mi hanno circondato», la VL, la Vg e Hier circumdederunt me $)^{55}$.

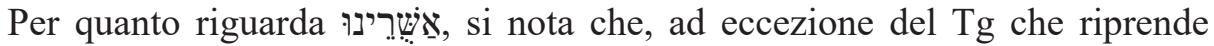
questo sostantivo, le restanti versioni antiche leggono una forma verbale, seguita sempre dal suffisso della 1a sg.: a) un participio (LXX, Sym, VL, Vg, Hier) subordinato in qualche modo [un'azione incoativa?] al ketib סָרבְוּנִ b) un verbo finito (Syr pf. Pael).

51 La terza possibilità porta a considerare to oggetto; cf. Van Uchelen, Psalmen I, 106: «Onze schreden ... nu omsingelen zij mij».

52 L'affermazione di Kissane, «Psalm XVII», 91, che of life")» (corsivo aggiunto), è incompleta, dal momento che l'uso proprio di che dal v.5, anche dai Sal 37,31; 40,3; 73,2.

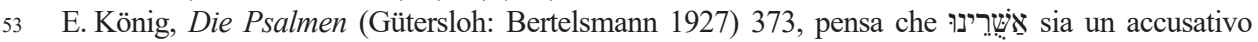
di relazione («In bezug auf unsern Schritt haben sie uns jetzt umringt») e rimanda per gli esempi a E. König, Historisch-comparative Syntax der hebräischen Sprache (Leipzig: Hinrichs 1897) $\S 328 \mathrm{f}$. Nel suo volume sulla sintassi ebraica, König esclude apparentemente il nostro brano: «aber in Ps $17_{11 \mathrm{a}}$ ist „,betreffs unseres Schrittes“ [...] nicht wahrscheinlich».

54 Si veda, ad es., la spiegazione assai complessa di E.F.C. Rosenmüller, Psalmi annotatione perpetua illustrati, 2a ed. (Scholia in Vetus Testamentum 4/1; Lipsiae: sumptibus Barthii 1821) 421: «Gressus nostros quod attinet, nunc circumdant nos, quocunque modo ego, Davides, cum sociis meis, gressus nostros dirigimus...».

55 Il ketib è preferito da J.B. de Rossi, Variae lectiones Veteris Testamenti (Parmae: ex Regio typographeo 1788) IV, 10 e da Kennicott, II, 317, che lo mette in corpore. 
La forma verbale di riferimento è riconducibile a due radici ebraiche: a) גרש,

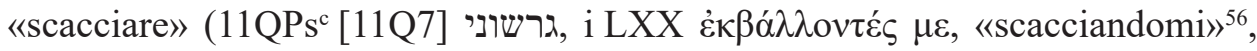
la VL e la Vg proicientes me); b) אשר con due significati diversi: $1^{\circ}$ «chiamare

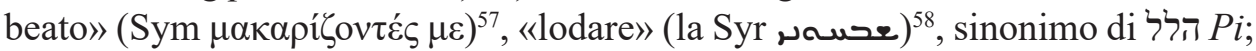
$2^{\circ}$ «avanzare contro» (Hier incendentes adversum me).

Queste due linee interpretative, che provengono dalla tradizione antica, sono possibili dal punto di vista critico-testuale ${ }^{59}$. La linea interpretativa che attinge a גרש mette bene in rilievo l'avversità contro l'orante e si addice al tenore del salmo, ma non ha avuto sviluppi nella tradizione manoscritta ebraica posteriore per almeno due motivi: a) $11 \mathrm{QPs}^{\mathrm{c}}$ (11Q7) è stato scoperto a Qumran fra il 1961 e il $1962^{60}$; b) i LXX hanno cessato d'essere un testo di riferimento per la comunità ebraica probabilmente verso la fine del I sec. d.C.

La scelta di rendere אשר con «chiamare beato» (Sym) o «lodare» (Syr) mal si accorda con i vv.9-12, dove un individuo si trova sotto l'attacco del nemico. Resta, quindi, la resa di Hier con la sua lettura più conforme al resto del salmo e in grado di risolverne la complessità interpretativa. Accogliere la lezione incendentes adversum me attestata probabilmente in un testo ebraico risalente ancora

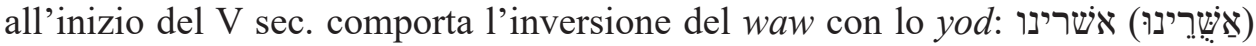

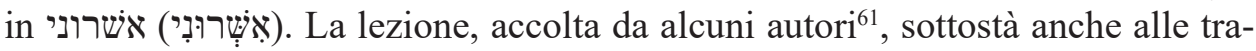
duzioni di Sym e della Syr (cf. Gn 30,13; Ml 3,12; Sal 41,3; 72,17; Gb 29,11;

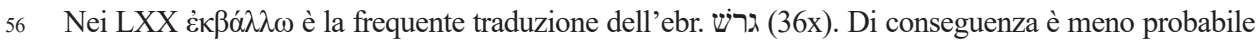

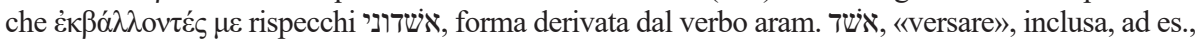
da $B H K, B H S$. La congettura ha però il merito di proporre una forma verbale seguita da un suff. della 1a sg. conforme al contesto, sebbene finisca per ipotizzare una doppia corruzione del TM (scambio di daleth con resh e di yod con waw). Anche se una traccia della sostituzione di resh con daleth è preservata in אשדינו del ms 89 (inizio del XIII sec.) di Kennicott, II, 317, restano prive di spiegazioni l'altro scambio e la stessa presenza di una forma aramaizzante. Invece, secondo F. Wutz,

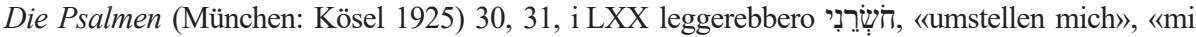
hanno accerchiato», nel senso di «inseguire», «dare a caccia». Abbiamo a che fare perciò con una ricostruzione congetturale dato che il verbo חשר non fa parte del lessico biblico.

57 In Pro 31,28, Sym rende אשר con $\mu \alpha \kappa \alpha \rho i ́ \zeta \omega$ (Qo 4,2 è discutibile). Questa resa trova più volte riscontro nei LXX (cf. Gn 30,13; Is 3,12; 9,15; Ml 3,12.15; Sal 40,3; 71,17; Gb 29,11; Ct 6,9).

58 Il ms 9a1 pone prima il dalet, mentre ms 10 t2 il waw. La Syr rende אשר con, «llodare», in Gn 30,13; Ml 3,12; Sal 17,11; 72,17; Gb 29,11.

59 Per a) גרש:II[/I] sec. a.C. (LXX) - prima metà del I sec. d.C. (11QPsc [11Q7]) - II-IV sec. (VL) - fine del IV sec. (Vg); per b) אשר : fine del II sec. (Sym) - II d.C. ca. (Syr dal proto-masoretico, cf. Carbajosa, «Peshitta», 93) - inizio del V sec. (Hier).

60 Cf. DJD 23, p. xiii.

61 Houbigant, Notae criticae, 11: «germana scriptio est אשרוני, incedunt in me». Senza commentare in dettaglio, questa lettura è stata adottata, ad es., da H. Herkenne, Das Buch der Psalmen (HSAT 5/2; Bonn: Hanstein 1936) 87: «Sie sind auf mich losgeschritten», e in seguito sia da G.R. Driver, «Notes on the Psalms. I. 1-72», JThS 43 (1942) 152: «they are hard on my tracks», sulla scorta dell'arabo ta 'attur, «followed the track of» e ripresa da Leveen, «Psalm XVII», 51, 53; sia da P.C. Craigie, Psalms I (1-50) (WBC 19; Waco, TX: Word Books 1983) 161; Lorenzin, I Salmi, 85; Barthélemy, Critique textuelle, 75: «Ils ont marché contre moi, maintenant ils m'entourent». 
Ct 6,9). Il mutamento delle consonanti, per un errore scribale ${ }^{62}$, spiegherebbe l'o-

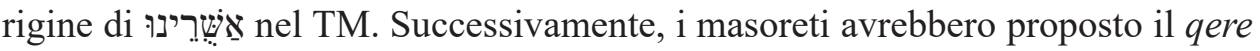

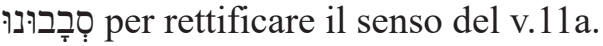

La nostra proposta resta evidentemente una congettura, poiché difficilmente se ne trova riscontro nella tradizione manoscritta ebraica, quantunque $B H K^{1-3}$ e $B H S$ citino un ms ebraico con אשרוני. Questa citazione scaturisce da un'annotazione di Giovanni Bernardo de Rossi ${ }^{63}$, secondo cui il ms 41 di Benjamin Kennicott legge אשרוני 41 (del 1475), ovvero nel ms Selden Super. 105, fol. 21r, della Bodleian Library ${ }^{65}$.

Alla luce delle riflessioni precedenti emerge la seguente variante del testo ebrai-

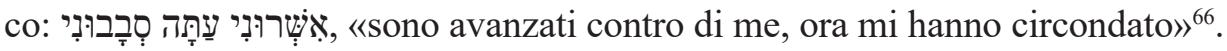

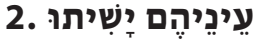

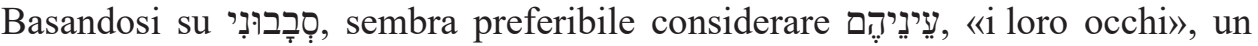

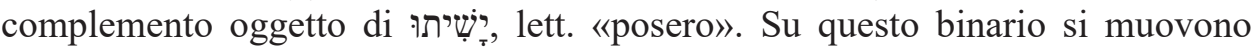

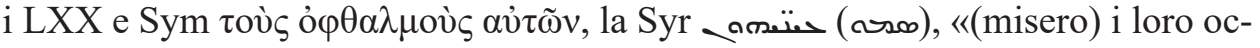

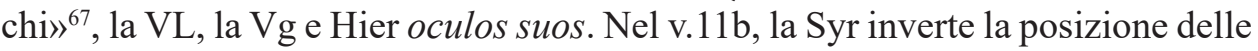
prime due parole per indicare chiaramente il complemento oggetto ed equipara

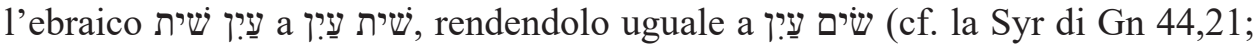
Ger 24,6; Am 9,4). Si nota anzitutto che il sintagma שית שעית שית שית è altrimenti sconosciuto nella Bibbia ebraica, mentre שים עַיטין ricorre sempre con la preposizione עַ, «porre gli occhi su qualcuno», «volgere lo sguardo su»; cf. Gn 44,21;

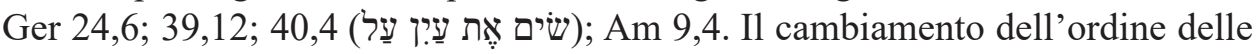
parole da parte della Syr suggerisce poi una certa indeterminatezza dell'originale ebraico. Per risolverla, va anzitutto mantenuta la sequenza dei vocaboli (che le versioni antiche succitate faticano a rispettare). In secondo luogo, occorre soffermarsi sullo stile del salmista. Quando un nome astratto o una parte del corpo funge da complemento oggetto, egli lo colloca ogni volta dopo il verbo finito (cf. vv.3a.4b.5b.14c). Al contrario, se è in funzione del soggetto, egli antepo-

62 Potrebbe essere intenzionale, in modo da continuare, ad es., una serie che menziona le parti del corpo (grassi, v.10a; bocche, v.10b; occhi, v.11b) oppure l'errore, tenendo in mente

63 De Rossi, Variae lectiones, IV, 10.

64 Kennicott, II, 317.

65 Cf. https://digital.bodleian.ox.ac.uk/objects/f700d242-b315-49d8-bcfa-bc49d009d8f7/surfaces/522bb467-9742-4f92-9c05-3ba766830f5f [accesso: 10 maggio 2021].

66 Alla luce di quanto detto in corpore, sono superflue le congetture di H. Gunkel, Die Psalmen, 4a

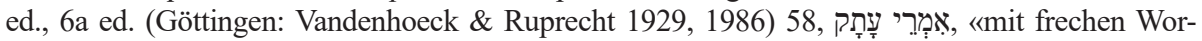
ten (umgeben sie mich)»; di Lindblom, «Bemerkungen», 9, אשרימו, «Jetzt umgeben mich ihre Schritte»; di H.-J. Kraus, Psalmen, 6a ed. (BKAT 15/1; Neukirchen-Vluyn: Neukirchener 1989)

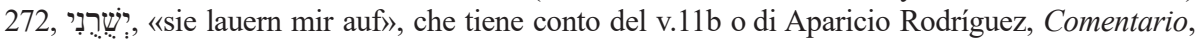

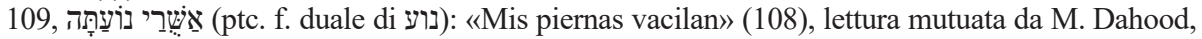
Psalms I (AncB 16; Garden City, NY: Doubleday 1966) 98.

67 Oppure «(e misero) i loro occhi», dal momento che alcuni mss pongono il waw prima di 
ne tale sostantivo al verbo finito (vv.2ab.3c.10ab.14b). Probabilmente עינינֶידם è il soggetto di יָּיָׁיתו. «I loro occhi (sogg.) guardavano per prostrare/stendere a terra» $\mathrm{o}$, in maniera più libera, «fissavano lo sguardo per stendermi a terra».

\section{Versetto 12}

simili a un leone che brama la preda, a un leoncello che si apposta in agguato.

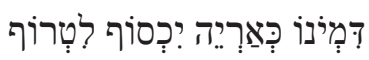

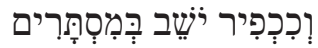

\section{1דִ}

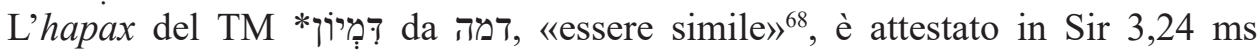
A דמיונות, «pensieri»e 1QM (1Q33) 6מיונים T,13, «somiglianze». Il significato di

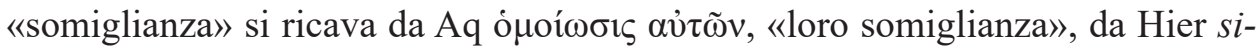
militudo eius e dal Tg דמותיה, "הsua somiglianza» (cf. la Syr, "Sono simili»)" Invece, per uniformità con il contesto precedente, in cui sono i nemici (non il nemico) a osteggiare l'orante, i LXX í $\tilde{\varepsilon} \lambda \alpha \beta \beta^{\prime} v \mu \varepsilon$, «mi considerarono» ${ }^{70}$ oppure «mi assalirono» ${ }^{71}$, la VL e la Vg susceperunt me, leggono una forma verbale (in

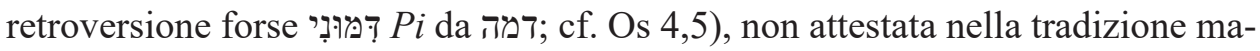
noscritta ebraica. Inoltre, il singolare «la sua somiglianza» (iֹịị) è responsabile del cambiamento dal plurale (vv.9-11) al singolare (v.12) ${ }^{72}$ che continua al v.13.

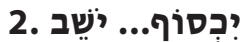

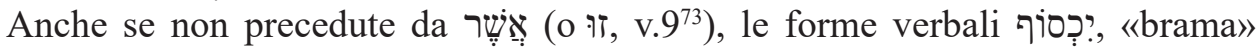

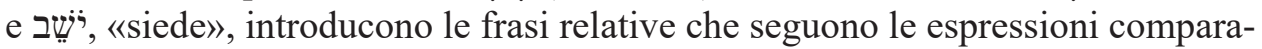

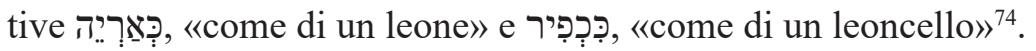

68 Cf. BL $\S 61 \mathrm{c} \theta . \mathrm{d} \theta$.

69 Cf. Gesenius Thes., 343; BDB, 198; HALAT, 217; Ges18, 254; DCHR II, 546. Invece, Zorell, 175 : «imaginatio, cogitatio, consilium [...] rei malae Ps $17_{12} »$.

70 Così Schleusner, III, 344: «existimarunt, cogitarunt apud se de me»; J. Lust-E. Eynikel - K. Hauspie, A Greek-English Lexicon of the Septuagint (Revised Edition) (Stuttgart: Deutsche Bibelgesellschaft 2003) 635: «they took me up, they thought of me»; A. Nicolotti, LXX-IT, 61: «Mi hanno considerato».

71 Così T. Muraoka, A Greek-English Lexicon of the Septuagint (Louvain - Paris - Walpole, MA: Peeters 2009) 703: «they came upon me»; E. Bons, LXX-D, 1535: «Sie überfielen mich».

72 Dal punto di vista grammaticale, il suff. i- ha probabilmente valore distributivo, riferendosi al gruppo dei nemici nei vv.9-11 (cf. Sal 63,11 in cui il suff. della 3a sg. m. si riferisce al gruppo del v.12); cf. Ibn Ezra דמיון כל אחד מהסובבים, «la forma di ciascuno di quelli che hanno circondato»; König, Wörterbuch, 71: «das Bild eines jeden von ihnen (3 § 348u [= Idem, Syntax]) gleicht»; si nota che König, nella propria sintassi, non cita il Sal 17,12.

73 î è l'unico pronome relativo usato nel Sal 17. Il suo carattere poetico si coglie in 17 delle sue 19 ricorrenze (ad eccezione di 1Re 6,1.37), di cui 11 nel Salterio; cf. GK § 138g; JM § 145c: «the wicked who have tormented me».

74 Cf. GK $§ 126$; Is 23,9 . 


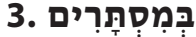

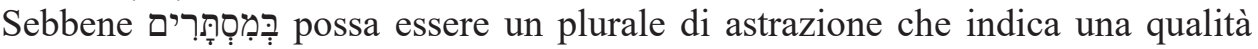
di azione: «in segreto», «di nascosto» (cf. Ger 13,17; Lm 3,10; cf. la Syr recob, «in segreto» $)^{75}$, è preferibile interpretare la frase in senso spaziale «in luoghi

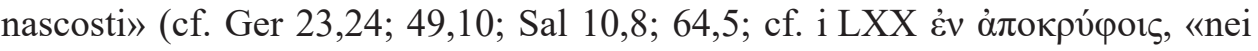
nascondigli», la VL e la Vg in abditis, «in posti segreti», Hier in absconditis, «in luoghi nascosti», il Tg בטומריא, «in luoghi nascosti»).

\section{Versetto 14}

con la tua mano, Signore, dai mortali, dai mortali del mondo, la cui sorte è in questa vita. Sazia pure dei tuoi beni il loro ventre, se ne sazino anche i figli e ne avanzi per i loro bambini.

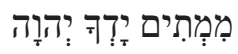

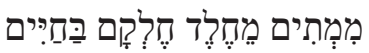

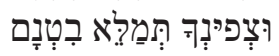

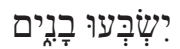

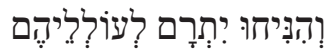

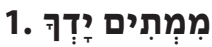

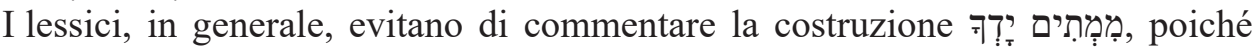
considerano il testo consonantico corrotto e la sintassi del v.14 problematica.

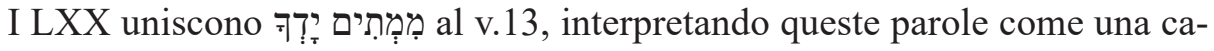

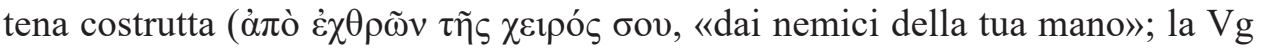
ab inimicis manus tuae; la VL de manu inimicorum; cf. 11QPs ${ }^{c}$ (11Q7) fr. 8:

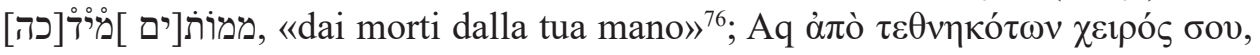
« מִמְתִים con ảंò ỏ $\lambda i ́ \gamma \omega v$, «dai pochi» (cf. la VL e la Vg a paucis), attribuendogli, in modo letterale, il significato secondario di מְ:, «uomini» ${ }^{77}$.

Tuttavia, ๆT? di mezzo («con la tua/per mezzo della tua mano») ${ }^{78}$. In questa direzione va

\section{Cf. JM $\S 136 \mathrm{~g}$.}

76 La fotografia di questo brano frammentario (cf. DJD 23, plate VI, fr. 8; https://www.deadseascrolls. org.il/explore-the-archive/image/B-298301 [accesso: 10 maggio 2021]) rivela che le lettere ות sono state ricostruite in basse a delle flebili tracce che non impongono la lettura di ות ma consentono anche il deciframento del solo $\Omega$; in tal caso avremmo una versione più vicina al TM.

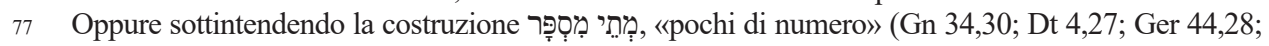

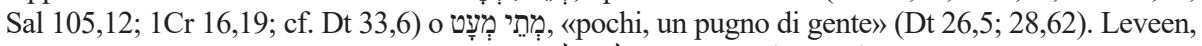

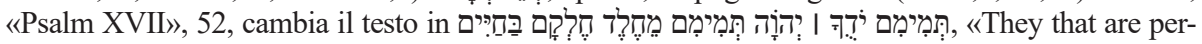
fect [sc. "in thy ways"] will praise thee, O Lord. As for the perfect of the world, their portion is in this life».

78 Per la mano come mezzo della realizzazione dell'opera divina, si vedano Dt 2,15; 7,19; Is 45,12; 49,22; Gb 19,21; cf. Sal 95,5; 119,73; Gb 10,8. 


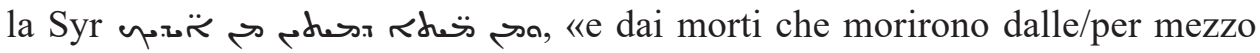
delle tue mani» (cf. 11Q7). Il nome («con la tua/di tua spada») $)^{79}$.

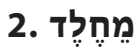

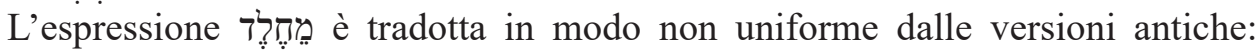

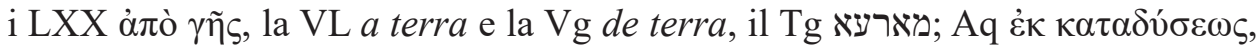

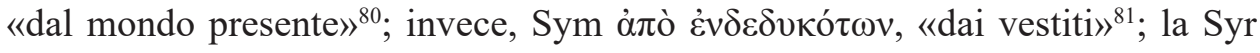
Кiqur, «della fossa/tomba»; Hier in profundo ${ }^{82}$. Benché l'uso in senso poetico del nome תֶֶ sia noto («mondo», «durata»; cf. Sal 39,6; 49,2; 89,48; Gb 11,17) ${ }^{83}$,

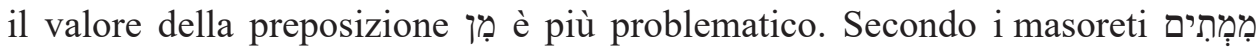
מִחֶל forma una frase, rispecchiata dalle traduzioni antiche ${ }^{84}$. Nel rispetto della punteggiatura masoretica, sono grammaticalmente possibili due soluzioni: il $\mathrm{min}$ privativo $^{85}$ o quello partitivo ${ }^{86}$. Nel primo caso, si tratterrebbe dei malvagi, definiti come uomini «privi di durata», quindi destinati a sparire subito ${ }^{87}$. È una soluzione che chiarisce la complessa frase ebraica. Essa non trova però riscontro nelle versioni antiche (LXX, Aq, Sym, VL, Vg, Tg) che appoggiano piuttosto il valore partitivo o separativo di מ̣ן. I malvagi sarebbero gli uomini concentrati sulle cose del mondo, che appartengono al mondo ${ }^{88}$, ossia dediti alle cose mondane e terrestri ${ }^{89}$.

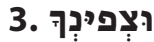

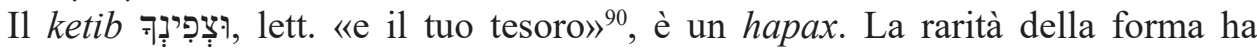
facilmente spinto i masoreti a proporre il qere di צפי, «nascosto», «serbato», «protetto», attestato ben cinque volte nella Bibbia (Ez 7,22; Os 13,12; Sal 83,4; Gb 20,26; Pro 13,22). Ad eccezione della Syr

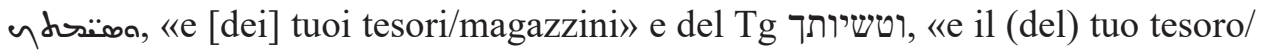

\footnotetext{
79 Per la spada come strumento della punizione divina, si vedano Dt 32,41; Sof 2,12; Zc 13,7.

80 In quanto soggetto al declino, poiché il primo significato di $\kappa \alpha \tau \alpha ́ \delta v \sigma 1 \varsigma$ è «immersione, discesa», «discesa nel sottosuolo», «tuffo» (cf. Sal 48,2 Sym).

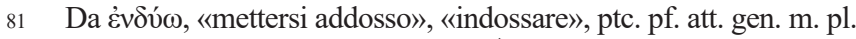

82 Il Sal 89,48 mostra che Hier legge חֶֶ,, «eternità», nel senso metaforico di «profondo», oppure sviluppa un significato secondario da «durata, mondo» $\rightarrow$ «profondo».

83 Si vedano, ad es., BDB, 317; HALAT, 303; Ges18, 351.

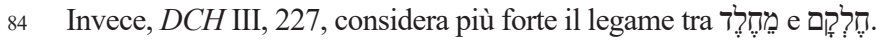

85 Cf. GK $\S 119 w$.

86 Cf. GK $§ 119 v ; J M ~ § 133 e$.

87 Parafrasando, Hier ha tradotto qui mortui sunt in profundo.

88 König, Wörterbuch, 108: «die [...] zur Welt gehören».

89 Kimchi, שהם מזה העולם, שכל תאותם וחפצם לזה העולם, (uomini) che sono di questo mondo, che ogni loro brama e desiderio è per il mondo»; Gesenius Thes., 474: «homines ex mundo i. e. rebus mundanis et terrestribus dediti, quare additur:

90 Gesenius Thes., 1181; BDB, 860; DCH VII, 149.
} 
magazzino», che a nostro avviso rispecchiano il ketib, il qere è stato accolto da

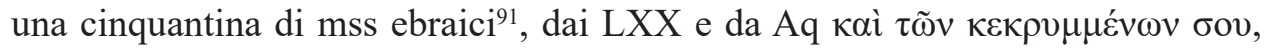
«e dei tuoi nascosti», seguiti dalla VL, dalla Vg e da Hier de absconditis tuis,

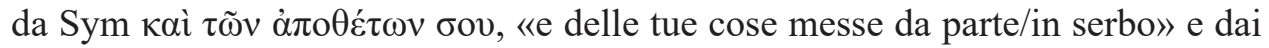
lessicografi moderni ${ }^{92}$. Il plurale, con cui le succitate versioni rendono il qere, non risolve definitivamente il problema relativo alla sua presenza nell'originale ebraico $^{93}$. Infatti, numerosi mss ebraici appoggiano il singolare (cf. Tg). Tuttavia, i LXX, Aq, Sym, la Syr, la VL, la Vg e Hier suggeriscono, se non altro, un'interpretazione collettiva ${ }^{94}$, rimuovendo l'incongruenza superficiale tra il singolare di qere/ketib e il plurale di

La traduzione del qere solleva un'altra questione relativa alla maniera in cui i vocabolari moderni interpretano l'insieme del v.14. Troviamo infatti «bene, proprietà $\rangle^{96}$, «risorse, mezzi nascosti $\rangle^{97}$, «doni migliori $\rangle^{98}$, «messo in serbo, riservato» ${ }^{99}$ (cf. Os 13,12; Gb 20,26), «(la cosa) a te destinata; ciò che hai in serbo» ${ }^{100}$, «magazzino, dispensa, tesoro» ${ }^{101}$, «tesoro» ${ }^{102}$ (cf. Ez 7,22; Pro 13,22); qualche lessicografo, poi, si astiene dal proporre una traduzione puntuale ${ }^{103}$. Si noti, in-

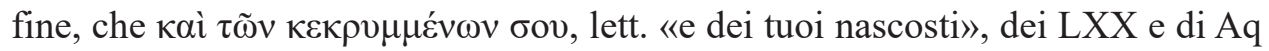

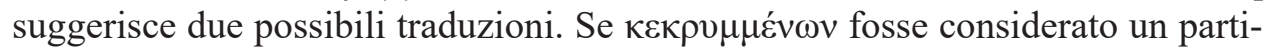
cipio neutro, significherebbe «beni nascosti» (abscondita; Abd 1,6; 2Mac 12,41; Mt 13,35; cf. Sym ${ }^{104}$; se, invece, fosse participio maschile, indicherebbe «(persone) protette» (absconditi; Dt 7,20; Gs 10,17) ${ }^{105}$. Quest'ultima resa è confermata

\footnotetext{
91 Cf. Kennicott, II, 317.

92 Cf. Gesenius Thes., 1180; BDB, 860; Gesenius, 693; König, Wörterbuch, 393; Zorell, 699; HALAT, 982; Ges18, 1133; DBHE, 642; DCH VII, 149.

93 Cf. וצפוניך nel ms 97 (inizio del XIV sec.) di Kennicott, II, 317. Per i LXX si tratta probabilmente

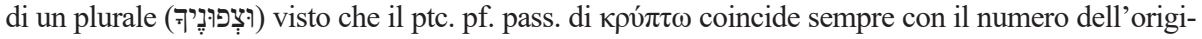
nale ebraico (Dt 7,20; Gs 10,17; Ez 12,6.7.12; Abd 1,6).

94 Così anche Craigie, Psalms, 161; F.-L. Hossfeld - E. Zenger, Die Psalmen. I. Psalm 1-50 (NEB; Würzburg: Echter 1993) 116; Lorenzin, I Salmi, 86, n. 15.

95 Attard, «From Well-fed Lions», 181-182, insiste, invece, sul reale spostamento quantitativo tra il singolare e il plurale.

96 König, Wörterbuch, 393: «Gut».

97 Gesenius Thes., 1180: «Part. pass. צִּפוּנִים opes reconditae».

98 Zorell, 699: «D[eu]s “optima dona" sua dat vel malis». La traduzione optima dona deriva dal fatto che il ptc. pass. indica una cosa custodita, un tesoro, un cimelio.

99 Gesenius, 693: «aufgespart».

100 HALAT, 982: «das von dir zugedachte».

101 DBHE, 642.

102 DCH VII, 149.

103 BDB, 860; Ges18, 1133.

104 Cf. Bons, LXX-D, 1536; Nicolotti, LXX-IT, 63.

105 Cf. Schleusner, II, 327.
} 
dal Sal 83,4 per il participio passivo Qal di צפן, «i tuoi protetti» (cf. i LXX $\tau \tilde{\omega} v$

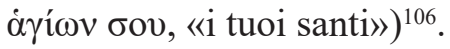

Raccogliendo i dati analizzati è possibile accettare il qere, considerandolo come sostantivo collettivo, con il significato di «e dei tuoi protetti». Questa frase, inoltre, introduce un nuovo pensiero del versetto, come risulta dalla presenza dell'accento

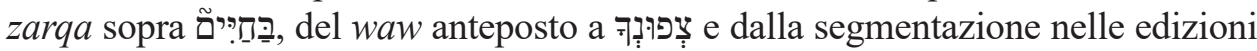
del testo biblico (Kennicott, $B H K^{1-3}, B H S, \mathrm{LXX}, \mathrm{Aq}, \mathrm{Sym}, \mathrm{VL}, \mathrm{Vg}$, Hier, Tg).

\section{Versetto 15}

Ma io nella giustizia contemplerò il tuo volto, al risveglio mi sazierò della tua immagine.

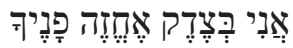

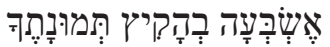

\section{1. אִחֶֶ}

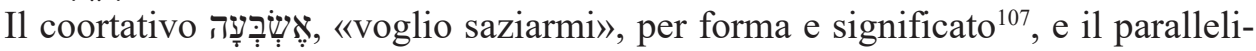
smo tra gli emistichi del v.15 gettano luce sullo yiqtol do di attribuirgli una sfumatura coortativa («voglio vedere»).

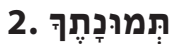

Il sostantivo con suffisso della seconda maschile singolare

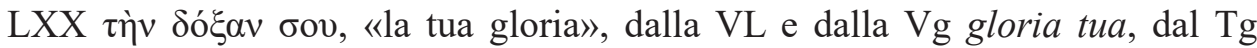

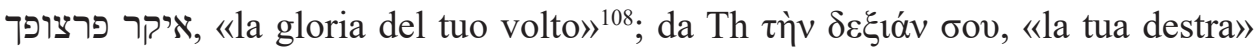
e dalla Syr y yrassm, «tua fede». La lezione masoretica va mantenuta, in quanto

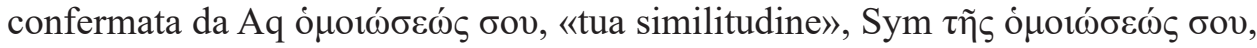
Hier similitudine tua e conforme al parallelismo con offrono le seguenti traduzioni: «figura», «forma», «somiglianza», «rappresentazione», «sembianza», «apparizione», «manifestazione» ${ }^{109}$. Forse la traduzione «sembianza» è da preferire, poiché unisce nel contempo la figura che appare

106 Il significato «tus protegidos» è adottato, senza ulteriori spiegazioni, da Aparicio Rodríguez, Comentario, 110, che tuttavia considera il waw come avversativo e traduce: «Pero llena el vientre de tus protegidos» (109); cf. Craigie, Psalms, 160, 161: «Your treasured ones»; Lorenzin, I Salmi, 86: «Ma ai tuoi protetti». Similmente, M. Mannati-É. de Solms, Les Psaumes (CPV; Paris: Desclée de Brouwer 1966) I, 187: «Tes bien-aimés», proponendo il senso derivato dal masoretico «et tes réserves (ou: tes protégés)». Leveen, «Psalm XVII», 53, legge il qere al plurale וּצְּפוּנִיך con l'accezione «and as for thy saints», mentre Dahood, Psalms I, 99, preferisce il ketib con la vocalizzazione defettiva di șepinek $\bar{a}$, «your treasured ones». Infine, R. Alter, The Hebrew Bible. III. Writings - Ketuvim. A Translation with Commentary (New York - London: Norton 2019) 54, che traduce: «And Your protected onesfill their bellies», commentando si tratta di coloro che sono nascosti sotto le ali divine (v.8).

107 Cf. JM $\S 114 \mathrm{c}$.

108 Si tratta di una correzione per motivi teologici.

109 Cf. Zorell, 901; HALAT, 1608; Ges18, 1443; DCH VIII, 640. 
senza contorni precisi ${ }^{110}$, il parallelismo con il «volto» del v.15a e ha una diffe-

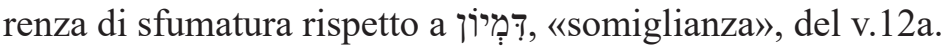

In una traduzione più libera, il v.15 potrebbe essere reso: «Quanto a me, nella giustizia voglio vedere il tuo volto! || Voglio saziarmi, al risveglio, della

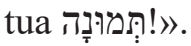

\section{Conclusione}

Lo studio del Salmo 17, ricco di tante sfumature lessicografiche ${ }^{111}$, è stato incentrato sul confronto critico-testuale tra il TM e le versioni antiche, senza dimenticare, tuttavia, altri testimoni ebraici. Fra i numerosi problemi discussi in questo articolo, alcuni meritano una menzione particolare:

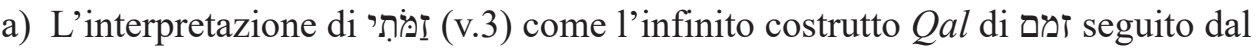
suffisso della 1a sg. con il senso de «il mio progetto»o «il mio pensiero», è stata preferita al perfetto Qal della $1 \mathrm{a} \mathrm{sg}$.

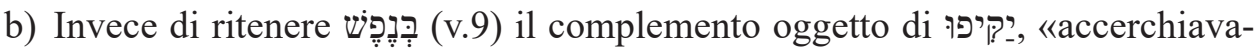
no» (cf. LXX, VL, Vg, Hier), la preposizione ᄁ̦̣ è stata considerata un complemento di modo e a נְֶ è stata attribuita la sfumatura di «bramosia».

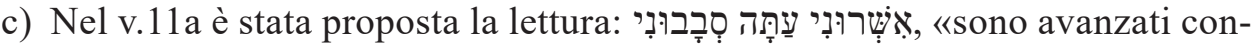

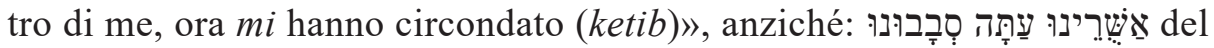
$\mathrm{TM}$, «i nostri passi, ora, ci hanno circondato (qere)». In questa occasione, è stato messo in evidenza che la citazione di un ms ebraico negli apparatus della $B H K$ e della $B H S$ a favore di damento.

d) Nel caso della crux del v.14, si è preferito mantenere integralmente la segmentazione del testo ebraico. È stata discussa, anche, la spinosa questione

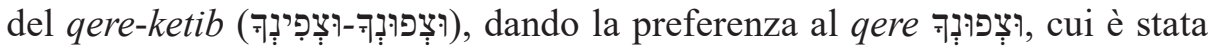
attribuita l'accezione collettiva di «e i tuoi protetti/custoditi».

e) Infine, per stabilire il valore preciso delle preposizioni ebraiche, occorre procedere con cautela nel ricorrere alle versioni antiche. Alle loro rese soggiacciono, infatti, delle ragioni stilistiche (cf. vv.2.4.9.14). In modo accessorio,

110 Cf. O. Loretz - P. Xella, «Beschwörung und Krankenheilung in RIH 78/20», Materiali lessicali ed epigrafici - I (Collezione di Studi Fenici 13; Roma: Consiglio Nazionale delle Ricerche 1982) 42; S. Schroer, In Israel gab es Bilder. Nachrichten von darstellender Kunst im Alten Testament (OBO 74; Freiburg: Universitätsverlag - Göttingen: Vandenhoeck \& Ruprecht 1987) 335-336; A. Favale, Dio d'Israele e dei popoli. Anti-idolatria e universalismo nella prospettiva di Ger 10,1-16 (AnBib.D 211; Roma: Gregorian \& Biblical Press 2016) 42.

111 Basterà ricordare che su un totale di 103 parole usate (inclusi il waw copulativo e le preposizioni), 65 parole ricorrono una volta, mentre 18 sono usate due volte. 
le traduzioni contribuiscono a determinare il senso delle preposizioni incluse nel Salmo 17 (cf. vv.1.4.9.14).

\section{Bibliografia}

Alonso Schökel, L., Diccionario bíblico hebreo-español, 2a ed. (Madrid: Trotta 1999) (= DBHE). Alter, R., The Hebrew Bible. III. Writings - Ketuvim. A Translation with Commentary (New York London: Norton 2019).

Aparicio Rodríguez, Á., Comentario filológico a los Salmos y al Cantar de los Cantares (Madrid: Biblioteca de Autores Cristianos 2012).

Attard, S.M., «From Well-fed Lions to Sitting Ducks. A Study of Complex Metaphors in Psalm 17», Networks of Metaphors in the Hebrew Bible (a cura di D. Verde - A. Labahn) (Bibliotheca Ephemeridum theologicarum Lovaniensium 309; Leuven - Paris - Bristol, CT: Peeters 2020) 173-191.

Barthélemy, D. et al., Critique textuelle de l'Ancien Testament. IV. Psaumes (Orbis biblicus et orientalis 50/4; Fribourg: Academic Press - Göttingen: Vandenhoeck \& Ruprecht 2005).

Bauer, H. - Leander, P., Historische Grammatik der hebräischen Sprache des Alten Testaments (Halle an d.S.: Niemeyer 1922) (= BL).

Baumgartner, W. et al., Hebräisches und aramäisches Lexikon zum Alten Testament (Leiden: Brill 1967-1996) I-V (= HALAT).

Biblia Sacra iuxta latinam Vulgatam versionem ad codicum fidem... cura et studio monachorum abbatiae pontificiae s. Hieronymi in Urbe ordinis sancti Benedicti edita. Liber Psalmorum (Romae: typis Polyglottis Vaticanis 1953) X.

Bons, E., «Psalm 16[17]», Septuaginta Deutsch. Erläuterungen und Kommentare zum griechischen Alten Testament (a cura di M. Karrer - W. Kraus) (Stuttgart: Deutsche Bibelgesellschaft 2011) 1534-1537 (= LXX-D).

Briggs, C.A. - Briggs, E.G., A Critical and Exegetical Commentary on the Book of Psalms (The International Critical Commentary; Edinburgh: Clark 1907) I.

Brown, F. - Driver, S.R. - Briggs, C.A., A Hebrew and English Lexicon of the Old Testament (Oxford: Clarendon 1906) (= BDB).

Cañas Reíllo, J.M., «Vetus Latina», The Hebrew Bible. I/C. Writings (a cura di A. Lange - E. Tov) (Textual History of the Bible 1; Leiden - Boston, MA: Brill 2017) 115-119.

Carbajosa, I., «Peshitta», The Hebrew Bible. I/C. Writings (a cura di A. Lange - E. Tov) (Textual History of the Bible 1; Leiden - Boston, MA: Brill 2017) 93-98.

Clines, D.J.A., The Dictionary of Classical Hebrew (Sheffield: Sheffield Academic Press 1996, 2001, 2007, 2010, 2011) III, V, VI, VII, VIII (=DCH).

Clines, D.J.A., The Dictionary of Classical Hebrew Revised (Sheffield: Sheffield Phoenix Press 2019) II (= DCHR).

Craigie, P.C., Psalms I (1-50) (Word Biblical Commentary 19; Waco, TX: Word Books 1983).

Dahood, M., Psalms I (The Anchor Bible 16; Garden City, NY: Doubleday 1966).

de Rossi, J.B., Variae lectiones Veteris Testamenti (Parmae: ex Regio typographeo 1788) IV.

Díez Merino, L. (a cura di), Targum de Salmos. Edición Príncipe del Ms. Villa-Amil n. 5 de Alfonso de Zamora (Bibliotheca Hispana Bíblica 6; Madrid: Consejo Superior de Investigaciones Científicas 1982). 
Driver, G.R., «Notes on the Psalms. I. 1-72», Journal of Theological Studies 43 (1942) 149-160.

Driver, G.R., «The Root פרץ in Hebrew», Journal of Theological Studies 25 (1924) 177-178.

Favale, A., Dio d'Israele e dei popoli. Anti-idolatria e universalismo nella prospettiva di Ger 10,1-16 (Analecta Biblica Dissertationes 211; Roma: Gregorian \& Biblical Press 2016).

García Martínez, F. - Tigchelaar, E.J.C. - van der Woude, A.S. (a cura di), Qumran Cave 11. II. 11Q2-18, 11Q20-30 (Discoveries in the Judaean Desert 23; Oxford: Clarendon Press 1998) (= DJD 23).

Gesenius, W., Hebräisches und aramäisches Handwörterbuch über das Alte Testament bearbeitet von F. Buhl, 17a ed. (Berlin - Göttingen - Heidelberg: Vogel 1962) (= Gesenius).

Gesenius, W., Hebräisches und aramäisches Handwörterbuch über das Alte Testament, 18a ed. (Berlin - Heidelberg: Springer 1987-2010) I-VI (= Ges18).

Gesenius, W., Thesaurus philologicus criticus linguae hebraeae et chaldaeae Veteris Testamenti, 2a ed. (Lipsiae: sumtibus typisque Vogelii 1829-1853) I-III (= Gesenius Thes.).

Gesenius, W. - Kautzsch, E., Hebräische Grammatik, 28a ed. (Leipzig: Vogel 1908) (= GK).

Glück, J.J., «Das Verb PRṢ in the Bible and in the Qumran Literature», Revue de Qumrân 5 (1964-65) 123-127.

Gunkel, H., Die Psalmen, 4a ed., 6a ed. (Göttingen: Vandenhoeck \& Ruprecht 1929, 1986).

Harland, P.J., «Robber or Violent Man? A Note on the Word pārîș», Vetus Testamentum 46 (1996) $530-534$.

Herkenne, H., Das Buch der Psalmen (Die Heilige Schrift des Alten Testaments 5/2; Bonn: Hanstein 1936).

Holmes, R. - Parsons, J., Vetus Testamentum Graecum cum variis lectionibus (Oxonii: e typographeo Clarendoniano 1823) III.

Hossfeld, F.-L. - Zenger, E., Die Psalmen. I. Psalm 1-50 (Neue Echter Bibel; Würzburg: Echter 1993).

Houbigant, C.-F., Notae criticae in universos Veteris Testamenti libros (Francofurti ad Moenum: apud Varrentrapp 1777) II.

Jenni, E., Die hebräische Präpositionen. I. Die Präposition Beth (Stuttgart-Berlin - Köln: Kohlhammer 1992).

Jenni, E., Die hebräische Präpositionen. III. Die Präposition Lamed (Stuttgart - Berlin - Köln: Kohlhammer 2000).

Joüon, P. - Muraoka, T., A Grammar of Biblical Hebrew (Revised Edition) (Subsidia Biblica 27; Roma: Editrice Pontificio Istituto Biblico 2006) (= JM).

Kennicott, B., Vetus Testamentum Hebraicum cum variis lectionibus (Oxonii: e typographeo Clarendoniano 1780) II (= Kennicott).

Kirkpatrick, A.F., The Book of Psalms. I. Psalms I-XLI (The Cambridge Bible for Schools and Colleges; Cambridge: University Press 1906).

Kissane, E.J., «Some Critical Notes on Psalm XVII», Biblica 9 (1928) 89-96.

König, E., Hebräisches und aramäisches Wörterbuch zum Alten Testament (Leipzig: Dieterich 1931).

König, E., Historisch-comparative Syntax der hebräischen Sprache (Leipzig: Hinrichs 1897).

König, E., Die Psalmen (Gütersloh: Bertelsmann 1927).

Kraus, H.-J., Psalmen, 6a ed. (Biblischer Kommentar Altes Testament 15/1; Neukirchen-Vluyn: Neukirchener 1989).

Leveen, J., «The Textual Problems of Psalm XVII», Vetus Testamentum 11 (1961) 48-54. 
Lindblom, J., «Bemerkungen zu den Psalmen I», Zeitschrift für die alttestamentliche Wissenschaft 59 (1942-1943) 1-13.

Lorenzin, T., I Salmi. Nuova versione, introduzione e commento (I Libri Biblici. Primo Testamento 14; Milano: Paoline 2000).

Loretz, O. - Xella, P., «Beschwörung und Krankenheilung in RIH 78/20», Materiali lessicali ed epigrafici - I (Collezione di Studi Fenici 13; Roma: Consiglio Nazionale delle Ricerche 1982) 37-46.

Lust, J. - Eynikel, E. - Hauspie, K., A Greek-English Lexicon of the Septuagint (Revised Edition) (Stuttgart: Deutsche Bibelgesellschaft 2003).

Mannati, M. - de Solms, É., Les Psaumes (Cahiers de la Pierre-qui-Vire; Paris: Desclée de Brouwer 1966) I.

Meade, J.M., «Hexaplaric Greek Translations», The Hebrew Bible. I/C. Writings (a cura di A. LangeE. Tov) (Textual History of the Bible 1; Leiden - Boston, MA: Brill 2017) 98-103.

Meyer, R., Hebräische Grammatik, 3a ed. (Sammlung Göschen 764/764a/764b; Berlin: De Gruyter 1969) II.

Muraoka, T., A Greek-English Lexicon of the Septuagint (Louvain - Paris - Walpole, MA: Peeters 2009).

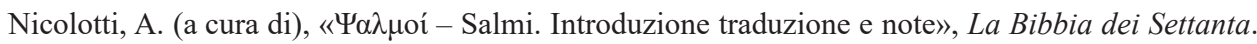
III. Libri poetici (a cura di C. Martone) (Antico e Nuovo Testamento 16; Brescia: Morcelliana 2013) 21-401 (= LXX-IT).

Oppenheim, A.L. et al., The Assyrian Dictionary (Chicago, IL: The Oriental Institute - Glückstadt: Augustin 1971, 1977) VIII, X/2 (=CAD).

van der Ploeg, J., «Le psaume xvii et ses problèmes», כה 1940-1965 (Oudtestamentische Studiën 14; Leiden: Brill 1965) 273-295.

Podechard, E., Le Psautier. Notes critiques. I. Psaumes 1-75 (Bibliothèque de la Faculté Catholique de Théologie de Lyon 4; Lyon: Facultés Catholiques 1949).

Rashi, Ibn Ezra e Kimchi, si veda M. Cohen (a cura di), Mikra'ot Gedolot 'Haketer': Psalms. I-II. A Revised and Augmented Scientific Edition (Ramat-Gan: Bar Ilan University 2003).

Rogers, J., «Vulgate», The Hebrew Bible. I/C. Writings (a cura di A. Lange - E. Tov) (Textual History of the Bible 1; Leiden - Boston, MA: Brill 2017) 104-110.

Rosenmüller, E.F.C., Psalmi annotatione perpetua illustrati, 2a ed. (Scholia in Vetus Testamentum 4/1; Lipsiae: sumptibus Barthii 1821).

Sabatier, P. (a cura di), Bibliorum sacrorum latinae versiones antiquae seu Vetus Italica (Parisiis: apud Franciscum Didot 1751) II.

de Sainte-Marie, H. (a cura di), Sancti Hieronymi Psalterium iuxta Hebraeos (Collectanea Biblica Latina 11; Roma: Abbaye Saint-Jérôme - Città del Vaticano: Libreria Vaticana 1954).

Schleusner, J.F., Novus thesaurus philologico-criticus: sive, lexicon in LXX et reliquos interpretes Graecos, ac scriptores apocryphos Veteris Testamenti, 2a ed. (Londini: Duncan 1829) I-III (= Schleusner).

Schroer, S., In Israel gab es Bilder. Nachrichten von darstellender Kunst im Alten Testament (Orbis biblicus et orientalis 74; Freiburg: Universitätsverlag - Göttingen: Vandenhoeck \& Ruprecht 1987).

von Soden, W., Akkadisches Handwörterbuch (Wiesbaden: Harrassowitz 1965, 1972, 1981) I-III $(=A H w)$. 
Smith, J., «Septuagint», The Hebrew Bible. I/C. Writings (a cura di A. Lange - E. Tov) (Textual History of the Bible 1; Leiden - Boston, MA: Brill 2017) 82-88.

Stec, D.M., «Targum», The Hebrew Bible. I/C. Writings (a cura di A. Lange - E. Tov) (Textual History of the Bible 1; Leiden - Boston, MA: Brill 2017) 88-93.

Stec, D.M. (a cura di), The Targum of Psalms (The Aramaic Bible 16; London - New York: Clark 2004).

Strawn, B.A., «Textual History of Psalms», The Hebrew Bible. I/C. Writings (a cura di A. Lange E. Tov) (Textual History of the Bible 1; Leiden - Boston, MA: Brill 2017) 5-23.

Toll, C., «Die Wurzel PRȘ in Hebräischen», Orientalia Suecana 21 (1972) 73-86.

van Uchelen, N.A., Psalmen. I. 1-40, 3 ed. (De prediking van het Oude Testament; Nijkerk: Callenbach 1986).

Waltke, B.K. - O'Connor, M., An Introduction to Biblical Hebrew Syntax (Winona Lake, IN: Eisenbrauns 1990) (=IBHSy).

Wutz, F., Die Psalmen (München: Kösel 1925).

Zorell, F., Lexicon Hebraicum et Aramaicum Veteris Testamenti (Romae: Pontificium Institutum Biblicum 1984) (= Zorell). 